\title{
Assessing the Impacts of Fractionation on Pointing-Intensive Spacecraft
}

\author{
Michael Gregory O'Neill ${ }^{*}$ and Annalisa L. Weigel ${ }^{\dagger}$ \\ Massachusetts Institute of Technology, Cambridge, MA, 02139
}

\begin{abstract}
Fractionated spacecraft consist of physically independent, "free-flying" modules composed of various subsystems. Thus, a fractionated spacecraft might consist of one module responsible for power generation and storage, another module responsible for communications, another module responsible for the payload, and so on. Fractionated spacecraft are of particular interest for pointing-intensive, remote sensing missions because of their ability to physically decouple subsystems and payloads that truly need precise pointing, thereby potentially enabling fractionated spacecraft to have a lesser lifecycle cost than that of a comparable monolithic spacecraft. This research seeks to explore this hypothesis and others by quantitatively assessing the impacts of various fractionated spacecraft architecture strategies on the lifecycle cost and mass of pointing-intensive, remote sensing mission spacecraft. A dynamic lifecycle simulation and parametric model was used to assess the lifecycle cost impacts, while the mass impacts were assessed using a nonparametric, physics-based computer model. Results from this research demonstrate that relative to a comparable monolithic spacecraft, fractionated spacecraft can have a lesser lifecycle cost but are always more massive.
\end{abstract}

ADS_GNS
Arch
Comm_CS_C\&DH
LCC
$M C A$
NRE
Power
RE
RSM(S)
SET
S/C-G

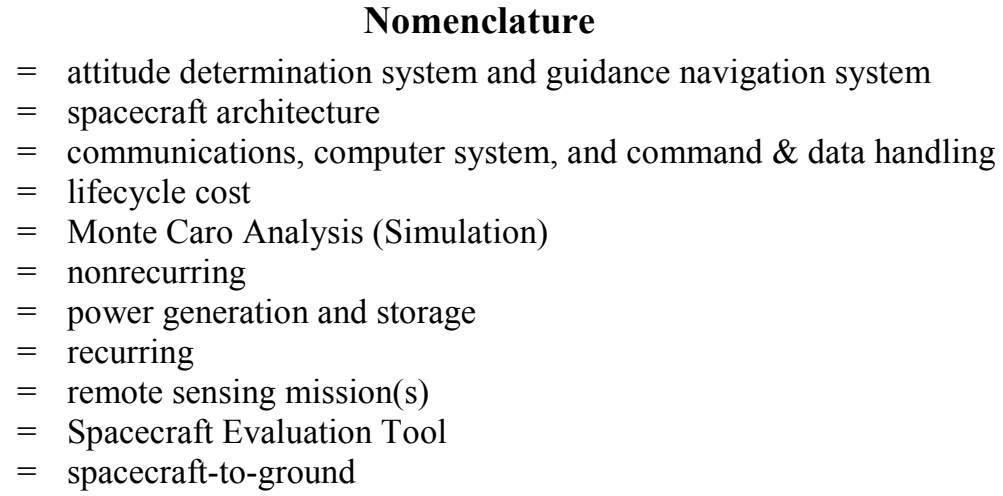

\section{Introduction}

$\mathrm{T}$ he hypothesis that fractionated spacecraft may be a suitable, if not "better" alternative to monolithic spacecraft for space missions has gained appreciable momentum in recent years. Instantiations both validating and invalidating this hypothesis have been demonstrated through previous assessments of fractionation; thereby, not providing a consistent argument as to whether or not fractionated spacecraft are a suitable alternative to monolithic spacecraft. However despite this inconsistency, these assessments of fractionation have provided meaningful motivation for the continued formulation of responses supporting and refuting this hypothesis. One aspect of this motivation originates from fractionated spacecraft purported as having diffuse benefits and concentrated costs, for a given space mission. The diffuse benefits are contended to be a function of fractionated spacecraft providing benefits not necessarily realized at the beginning of a lifecycle, but rather that emerge over the course of the lifecycle. And the concentrated costs are predominantly alleged to be a result of large upfront costs associated with developing and deploying fractionated spacecraft.

\footnotetext{
${ }^{*}$ Graduate Research Assistant, Aeronautics and Astronautics, Bldg. E38-550, and Student Member AIAA.

${ }^{\dagger}$ Assistant Professor, Aeronautics and Astronautics, Bldg. 33-404, and AIAA Member.
} 
The purported concentrated costs of fractionated spacecraft serve as the fundamental source of motivation for this research investigation, through which responses are formulated to the hypothesis regarding the (un)suitability of fractionated spacecraft in the current spacecraft paradigm. Specifically, this research investigation assesses the value propositions (e.g., lifecycle cost and mass) of pointing-intensive monolithic and fractionated spacecraft in the context of remote sensing missions (RSMs), thereby offering a quantitative perspective for understanding the implications of fractionated spacecraft yet to be enumerated in other assessments of fractionation.

\section{A. Fractionated Spacecraft}

Fractionated spacecraft consist of a set of structures (referred to as modules) that are physically independent and often collaborate in some capacity to attain a particular level of performance during a space mission. This definition for fractionated spacecraft leads them to have the same connotation as distributed spacecraft; however, the term fractionated is adopted and used herein. Additionally, this definition of fractionated spacecraft necessarily differentiates them from modular spacecraft, as the latter can have physically dependent constituents (Baldwin \& Clark, 1997; Esper, 2005; Esper et al., 2004; O'Neill, 2009). The modules in a fractionated spacecraft are meant to maintain either a cluster flying or formation flying on-orbit configuration; cluster flying requires less stringent relative positioning amongst modules than does formation flying. The on-orbit configuration shape of a fractionated spacecraft's respective modules is most often envisioned as a "central" module surrounded by the remaining modules, which assume the position of evenly spaced points on an imaginary circle around, and a certain distance away from, the central module (akin to a ring around a bullseye).

The modules in a fractionated spacecraft are composed of various subsystems and each module does not necessarily have the same subsystem/hardware composition as the other modules (C. Mathieu \& Weigel, 2005a; O'Neill, 2009). Additionally, the modules in a fractionated spacecraft can collaborate to share certain subsystem resources and, although sometimes subtle, collaboration amongst modules is one of the key attributes of, and areas of technology development for, fractionated spacecraft. Through the creation of modules and subsequent sharing of resources via collaboration, fractionated spacecraft can physically decouple the pointing-intensive payload(s) and subsystems from the other subsystems/modules. Subsequently, this ability of fractionated spacecraft makes them of particular interest for remote sensing missions because it is hypothesized that this will enable them to have a lesser lifecycle cost than comparable monolithic spacecraft.

\section{B. Sharing Subsystem Resources}

Fractionated spacecraft physically decouple (i.e., separate) subsystems and payloads by placing them on different modules and, in doing so, are able to share certain subsystem resources amongst modules (Brown \& Eremenko, 2006a). For example, one module may not have a dedicated communications subsystem (decreasing its respective communications related requirements) and therefore must rely on collaborating with another module that has a dedicated communications subsystem (increasing its respective communications related requirements). Subsequently, through the dispersion and sharing of subsystem resources in a fractionated spacecraft, there is associated hardware required on the modules in the fractionated spacecraft providing the shared resources (aka sources) as well as those modules that rely on and receive shared resources (aka recipients). Following on the previous example in sharing the communications subsystem, the source(s) would require (1) a dedicated spacecraftto-ground (S/C-G) antenna, (2) one inter-module relay antenna for each recipient module in the fractionated spacecraft, and (3) the hardware directly associated with these antennas (e.g., a diplexer, radio frequency switch, low pass filter, transponder, band-reject filter, and multiplexer). In contrast, the recipient(s) of the communications resource would (1) no longer require a dedicated S/C-G antenna and instead only require (2) one inter-module relay antenna for inter-module communication, and (3) its associated hardware. The sharing of resources therefore necessarily requires the wireless interaction (i.e., collaboration) amongst modules in a fractionated spacecraft due to their physically independent nature and possible need to replenish modules during the spacecraft's lifecycle.

This research investigation considers the employment of three classes (domains) of shared subsystem resources amongst modules in a fractionated spacecraft: (1) communications, computer system, and command \& data handling (Comm_CS_C\&DH) (Brown \& Eremenko, 2006a); (2) attitude determination system and guidance navigation system (ADS_GNS) (Mandy, Sakamoto, Saenz-Otero, \& Miller, 2007; McGhan, Besser, Sanner, \& Atkins, 2006); and (3) power generation and storage (Power) (Kerslake, 2008; O'Neill, 2009). Given present space-qualified subsystem technologies, these three classes of shared resources appropriately encapsulate the current potential to share subsystem resources amongst modules in a fractionated spacecraft. The motivation for considering the use of shared resources is that they are necessary for fully realizing the benefits to be had from employing fractionation to decouple the payload(s) and subsystems in a spacecraft that truly require precise pointing from those subsystems that do not. 


\section{Earth-based, Pointing-intensive, Remote Sensing Missions}

Earth-based remote sensing missions (RSMs) make observations of the Earth over a specific range or ranges of the electromagnetic spectrum; often the range(s) is(are) in the visible, infrared, and near-infrared portions of the electromagnetic spectrum. The RSM observations reflect the particular Earth coverage statistics required by the mission at hand and can include observing all/part of Earth's surface, oceans, atmosphere, magnetosphere, weather, resources, health of crops, and/or pollution. This research investigation considers spacecraft performing RSMs with the objective of capturing visible wavelength images of the Earth's surface. The spacecraft therefore must have an optical mirror system (i.e., telescope) as their payload instrument and, for the purposes of this research, these spacecraft will have an orbit altitude and inclination of $700 \mathrm{~km}$ and $98^{\circ}$ respectively; a common altitude and inclination for past and present Earth-based RSMs (consider GeoEye-1, Landsat-7, and EOS Aqua).

The Earth-based, RSM spacecraft considered herein are also pointing-intensive due to their low pointing error (i.e., high pointing accuracy) as is necessarily required by their respective high performance payloads that can capture Earth images at high resolutions (e.g., 0.5 and $1 \mathrm{~m}$ ). Subsequently, pointing-intensive spacecraft have low pointing tolerances (i.e., allowable pointing error) of around 36 milli-arcseconds (1e-4 $4^{\circ}$. Pointing error in a spacecraft is the difference between the desired pointing direction and the actual pointing direction (i.e., spacecraft pointing axis), relative to a (spacecraft) inertial frame of reference.

\section{Literature Review}

Previous assessments of fractionation have been conducted in academia, industry, and government, each of which appreciably contributed knowledge towards an understanding of fractionated spacecraft. In academia, research efforts have predominantly been conducted at the Massachusetts Institute of Technology beginning in 2001 and continuing today. The majority of these research efforts have focused on quantitatively assessing the benefits and costs of fractionated spacecraft through the metrics of utility and static lifecycle cost, respectively (Diller, 2002; C. Mathieu \& Weigel, 2005a, 2005b, 2006; Charlotte Mathieu, 2006; Ross, 2006, 2003). However, industry and government provided the first known study of fractionation, which was published in 1984; but then it was not until 2004 that further publications pertaining to fractionated spacecraft emerged from the industry and government sector (Molette, Cougnet, Saint-Aubert, Young, \& Helas, 1984). In industry and government, the majority of the research pertaining to fractionated spacecraft has been conducted and/or led by Owen Brown. And to date, the most significant and recent assessment of fractionation is being conducted through the Defense Advanced Research Projects Agency (DARPA) System Future, Fast, Flexible, Fractionated, Free-Flying (F6) Program (Brown, 2004; Brown \& Eremenko, 2008; Brown, Eremenko, \& Roberts, 2006; Brown, Long, Shah, \& Eremenko, 2007; Defense Advanced Research Projects Agency, 2008; Shah \& Brown, 2008).

\section{E. Research Motivation: Problem Statement and Contributions}

To ensure the respective outcomes of this research effort provide a meaningful contribution to the greater spacecraft community with regard to understanding fractionation, three crucial limitations of the research efforts enumerated in the literature review were identified. Subsequently, these limitations were used to form the problem statement for this research. These three limitations, of which each previous assessment of fractionation has at least one of, are based on the specific objectives of, and resources allocated for, this research investigation - not the respective objectives of, and resources allocated for, previous assessments of fractionation. The first of these limitations is the use of low fidelity models (e.g., parametric or design-by-analogy models) coupled with limited or no consideration of sharing subsystem resources in fractionated spacecraft. The second limitation is the lack of dynamic lifecycle considerations, thereby meaning that the costs and benefits of fractionated spacecraft are assessed assuming a perfect lifecycle (i.e., there are no launch vehicle or on-orbit failures; subsequently, there is no need to replenish spacecraft /modules throughout the lifecycle). And the third limitation is a minimal focus of cardinal measures of benefit and/or value which is readily apparent by a reliance upon the metric of utility for quantifying the benefits (or lack thereof) of fractionated spacecraft. Therefore the problem statement for this research investigation and its respective contributions are:

Given the limitations of previous assessments of fractionated spacecraft, there is a need for...

\section{Research Contributions}

1. high-fidelity, dynamic quantitative assessments of fractionated spacecraft value propositions;

2. an understanding of fractionated spacecraft value propositions using cardinal, "traditional" measures of effectiveness (MoE);

3. and an exploration of fractionated spacecraft value propositions in both breadth and depth. 


\section{Methodology: The Spacecraft Evaluation Tool}

The Spacecraft Evaluation Tool (SET) was developed to perform the quantitative assessments of monolithic and fractionated spacecraft value propositions for this research investigation. The SET is embodied in a software program that uses a Microsoft Excel ${ }^{\circledR}$ and Matlab ${ }^{\circledR}$ integrated programming language platform and graphical user interface (GUI). The SET was designed and built (i.e., programmed) from the "ground up" thereby not relying, in any capacity, on models developed by others; the only exception being certain elements of the SET cost model as will be enumerated later. The three distinct functional divisions of the SET are inputs, model processes (simulation), and outputs. The SET employs a Monte Carlo Analysis (MCA) to simulate the potential dynamic lifecycle's for a given spacecraft based on the specified SET input values, and subsequently, generates a set of outputs (i.e., the SET outputs) to form the value proposition for that spacecraft. The SET functional flow is given in Figure 1 whereby the major inputs and outputs of the SET are provided as well as the highest (most conceptual) SET model processes.

\section{A. SET Inputs}

The SET inputs provide adequate metrics (degrees of freedom) to enable sufficient breadth in exploring value propositions for pointingintensive, RSM spacecraft, while observing the need to keep the model complexity tractable. The SET inputs thereby embody part of the third research contribution. In terms of spacecraft value propositions, the SET inputs can conveniently be thought of as "knobs" that can be "turned" and in doing so, within the confines of RSMs, completely transform the context in which spacecraft operate and their respective value

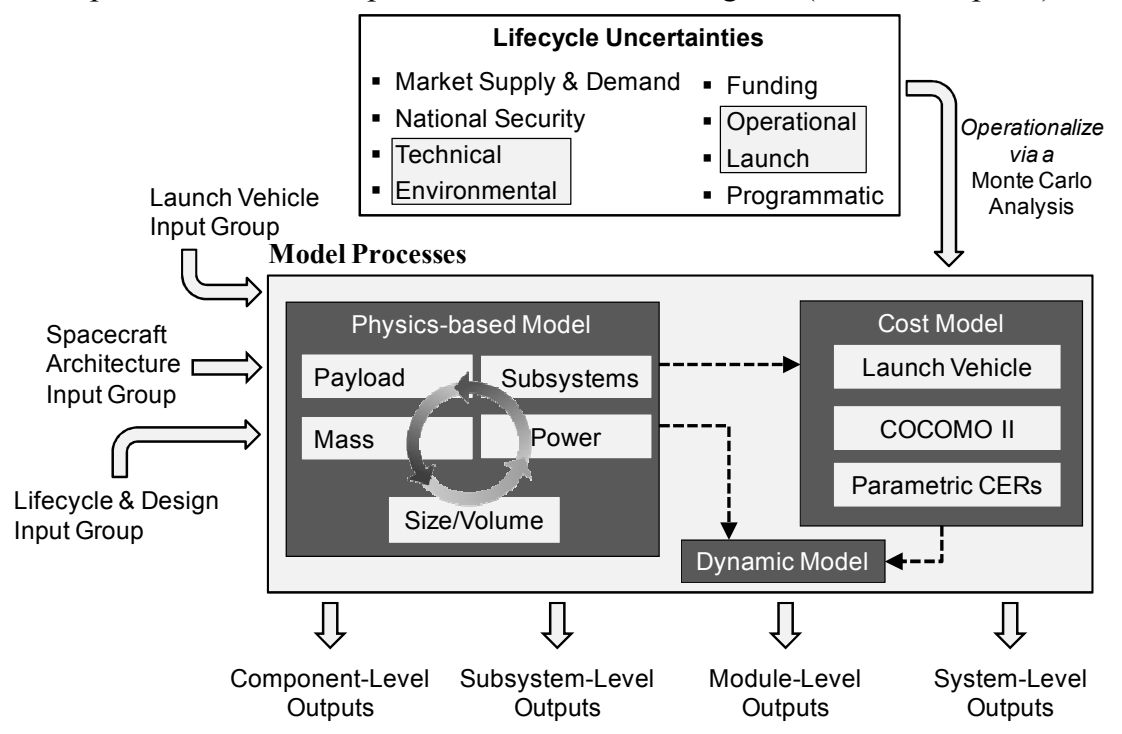

Figure 1. SET Overview: Functional Flow Block Diagram

propositions, for better or worse. A given spacecraft simulation (assessment) performed by the SET requires 154 independent input values to be specified, which subsequently form the context for quantifying the value proposition. Each of the 154 SET inputs is categorized as belonging to one of three input groups: Launch Vehicle (22 inputs), Lifecycle \& Design (20 inputs), or Spacecraft Architecture (112 inputs).

\section{Launch Vehicle}

The Launch Vehicle group of SET inputs specifies the launch vehicles that can be used for initial spacecraft/module deployment as well as spacecraft/module replenishments throughout the lifecycle. Presently, the SET allows for the selection of up to 22 launch vehicles that can be used during a spacecraft's respective lifecycle. The 22 launch vehicles span the small, medium, large, and heavy launch vehicle classes, which correspond to a max push mass to LEO of $<1000,1000-3000,3000-7000,>7000 \mathrm{~kg}$, respectively. In terms of international representation, of the 22 launch vehicles, 11, 8, 1, 1, and 1 are from the United States, Russia \& Ukraine, Italy \& Europe, China, and India respectively. The pertinent information for each respective launch vehicle, as is required for the launch vehicle selection model embedded in the SET, includes launch vehicle cost, stage masses/mass fractions, payload fairing dimensions, launch site latitude, and reliability (success rate). The launch vehicle data was obtained directly from launch vehicle manufacturers if possible and, if not, from Steven Isakowitzs' International Reference Guide to Space Launch Systems (Isakowitz, Hopkins, \& Hopkins Jr., 2004).

2. Lifecycle \& Design

The Lifecycle \& Design group of SET inputs, of which there are 20, define lifecycle (mission) context in which a given monolithic or fractionated spacecraft operates as well as certain parameters governing the design of these spacecraft. The 20 Lifecycle \& Design inputs are grouped into 11 categories: (1) orbital parameters (e.g., orbit altitude and inclination); (2) concept of operations (e.g., inter-module separation distance); (3) autonomy level (for bus and payload processing); (4) mission lifetime (for spacecraft lifecycle); (5) sizing (e.g., minimum launch vehicle packing efficiency); (6) payload performance (e.g., ground resolution); (7) pointing requirements for the pointing- 
intensive and non-pointing-intensive spacecraft/modules; (8) dynamic lifecycle simulation (e.g., number of MCA trials, probability of infant mortality); (9) production (e.g., learning factors); (10) lifecycle uncertainties (e.g., launch and technical) ${ }^{1}$; and (11) launch vehicles (e.g., number of simultaneous launches allowed for deployments).

3. Spacecraft Architecture ${ }^{2}$

The Spacecraft Architecture group of SET inputs comprehensively defines the monolithic and fractionated spacecraft architectures (designs) to be assessed by the SET. To fully define, that is, build a given spacecraft architecture, 112 spacecraft architecture-related inputs must be specified which include: (1) the number of modules; and then for each module (2) the subsystem composition, (3) the use of shared resources (i.e., is the module a shared resource source or recipient), (4) whether it has a RSM payload, and (5) whether it has a S/C-G directional antenna. As such, each spacecraft is built from the "ground up" (i.e., component-by-component, subsystem-by-subsystem, and module-by-module). The fidelity of the SET therefore enables the discrimination and subsequent understanding of nuances in monolithic and fractionated spacecraft architectures down to the component-level (sub-subsystem level). For a given spacecraft, if a module contains a RSM payload it is referred to as a payload module, otherwise it is referred to as an infrastructure module.

\section{B. SET Model Processes}

The SET model processes transform the SET inputs into the outputs via a simulation that consists of three models: physics-based, cost, and dynamic. Each model may contain several high-level model processes that in turn contain a multitude of sub-processes and sub-sub-processes, etc. not enumerated herein. However despite this, the three SET models and their respective model processes, as characterized by the design structure matrix (DSM) ${ }^{3}$ in Figure 2, provide a succinct conceptual overview of the "inner workings" (flow of information) in the SET for a given simulation. The confluence of the three models in the SET engender all three research contributions, namely, the creation of a high fidelity, dynamic spacecraft model that, given the SET inputs, can explore spacecraft value propositions, comprised of cardinal measures of effectiveness, in both breadth and depth.

1. Physics-based Model

To be capable of accurately modeling the spacecraft architectures specified by the SET inputs, the SET needed to be a high-fidelity spacecraft model. Subsequently, no aspect of the physics-based model relies on parametric models. The physicsbased model specifically consists of ten model processes as shown in Figure 2, each of which contains numerous smaller scope

processes that are not enumerated herein to keep the discussion of the physics-based model tractable. The physics-based model development minimized

\begin{tabular}{|c|c|c|c|c|c|c|c|c|c|c|c|c|c|c|c|c|c|c|}
\hline Model & & Model Process & 1 & 2 & 3 & 4 & 5 & 6 & 7 & 8 & 9 & 10 & 11 & 12 & 13 & 14 & 15 & 16 \\
\hline Inputs & 1 & SET Inputs & & & & & & & & & & & & & & & & \\
\hline \multirow{10}{*}{$\begin{array}{c}\text { Physics- } \\
\text { Based } \\
\text { Model }\end{array}$} & 2 & RSM Payload & $\mathrm{X}$ & & & & & & & & & & & & & & & \\
\hline & 3 & Computer System, C\&DH & $\mathrm{X}$ & $\mathrm{X}$ & & & & & & & & & \multirow{2}{*}{\multicolumn{4}{|c|}{$\begin{array}{c}\text { Feedback } \\
\text { Loops }\end{array}$}} & & \\
\hline & 4 & Communications, TT\&C & $\mathrm{X}$ & & $\mathrm{X}$ & & & & & & & & & & & & & \\
\hline & 5 & ADS, GNS & $\mathrm{X}$ & & & & & & & & & & & & & & & \\
\hline & 6 & EPS & $\mathrm{X}$ & & & & & & & & $\mathbf{X}$ & & & & & & & \\
\hline & 7 & Propulsion, ACS, GCS & $\mathrm{X}$ & & & & & $\mathrm{X}$ & & & & $\mathbf{X}$ & $\mathbf{X}$ & & & & & \\
\hline & 8 & TCS & $\mathrm{X}$ & $\mathrm{X}$ & $\mathrm{X}$ & $\mathrm{X}$ & $\mathrm{X}$ & $\mathrm{X}$ & $\mathrm{X}$ & & & & $\mathbf{X}$ & & & 7 & & \\
\hline & 9 & Power Required & $\mathrm{X}$ & $\mathrm{X}$ & $\mathrm{X}$ & $\mathrm{X}$ & $\mathrm{X}$ & $\mathrm{X}$ & $\mathrm{X}$ & $\mathrm{X}$ & & $\mathbf{X}$ & $\mathbf{X}$ & & & & & \\
\hline & 10 & Mass & $\mathrm{X}$ & $\mathrm{X}$ & $\mathrm{X}$ & $\mathrm{X}$ & $\mathrm{X}$ & $\mathrm{X}$ & $\mathrm{X}$ & $\mathrm{X}$ & & & & & & & & \\
\hline & 11 & Size, Volume & $\mathrm{X}$ & $\mathrm{X}$ & $\mathrm{X}$ & $\mathrm{X}$ & $\mathrm{X}$ & $\mathrm{X}$ & $\mathrm{X}$ & $\mathrm{X}$ & & $\mathrm{X}$ & & & & & & \\
\hline & 12 & LV Selection & $\mathrm{X}$ & $\mathrm{X}$ & $\bar{X}$ & $\mathrm{X}$ & $\mathrm{X}$ & $\mathrm{X}$ & $\mathrm{X}$ & $\mathrm{X}$ & & $\mathrm{X}$ & $\mathrm{X}$ & & & & & \\
\hline Cost & 13 & COCOMO II & $\mathrm{X}$ & $\mathrm{X}$ & & & & & & & & & & & & & & \\
\hline & 14 & Parametric CERs & $\mathrm{X}$ & $X$ & $\mathrm{X}$ & $\mathrm{X}$ & $\mathrm{X}$ & $\mathrm{X}$ & $\mathrm{X}$ & $\mathrm{X}$ & & $\mathrm{X}$ & & & & & & \\
\hline Dynamic & 15 & Lifecycle Simulation (MCA) & $\mathrm{X}$ & & & & & & & & & $\mathrm{X}$ & $\mathrm{X}$ & $\mathrm{X}$ & $\mathrm{X}$ & $\mathrm{X}$ & & \\
\hline Outputs & 16 & SET Outputs & & $\mathrm{X}$ & $\bar{X}$ & $\mathrm{X}$ & $\mathrm{X}$ & $\mathrm{X}$ & $\mathrm{X}$ & $\mathrm{X}$ & $\mathrm{X}$ & $\mathrm{X}$ & $\mathrm{X}$ & $\mathrm{X}$ & $\bar{X}$ & $\mathrm{X}$ & $\mathrm{X}$ & \\
\hline
\end{tabular}

Figure 2. SET Model Processes: Design Structure Matrix (DSM). Notation: command \& data handling (C\&DH); telemetry, tracking, \& control (TT\&C); attitude determination and guidance determination system (ADS, GNS); electric power system (EPS); attitude and guidance control system (ACS, GCS); launch vehicle (LV); cost estimating relationships (CERs); and Monte Carlo Analysis (MCA) the number of feedback loops to six; the feedback loops are addressed in the SET by employing an under and overrelaxed iterative procedure analogous to those employed in the field of Computational Fluid Dynamics for the convergence of flow field solutions (Lack, 2006).

\footnotetext{
${ }^{1}$ The dynamic simulation of spacecraft lifecycle's performed within the SET accounts for risks resulting from launch, technical, environmental, and operational lifecycle uncertainties, all of which adversely affect a spacecraft's respective performance (Brown \& Eremenko, 2006b; McManus \& Hastings, 2006; O'Neill, 2009). To mimic a spacecraft's naturally stochastic lifecycle, these lifecycle uncertainties affect a spacecraft "randomly" based on statistical distributions defined by the SET inputs. Subsequently, the probability of occurrence for the risks resulting from lifecycle uncertainties is never constant in a simulation.

${ }^{2}$ The term architecture directly follows spacecraft to emphasize structural (i.e., subsystem and hardware) differences.

${ }^{3}$ DSM: The X's in a given row represent the inputs required for the model process on that respective row, whereas the X's in a given column represent outputs from the model process in that respective column to other model processes.
} 


\section{Cost Model}

For a given launch vehicle payload (i.e., module or set of modules) and the launch vehicles permitted for use, the launch vehicle selection model process selects between one and three launch vehicles that can collectively fit the launch payload constituents, both in terms of the launch vehicle payload mass and physical dimensions given the destination orbit and launch site latitude(s). The criteria for the launch vehicle(s) selection are to minimize and maximize the aggregate launch vehicle cost and reliability respectively. The launch vehicle selection model process therefore mimics the behavioral characteristics of a cost and risk-averse individual.

The remaining two model processes in the cost model are the COCOMO II and parametric cost estimating relationships (CERs). Given the proprietary nature of industry-employed spacecraft cost models and the general lack of cost information available from spacecraft hardware manufacturers, it was unrealistic to develop a comprehensive bottom-up (high fidelity) cost model to match the fidelity of the physics-based model. Subsequently, the cost model employs (1) COCOMO II for the computer cost and software development time estimation; (2) massbased CERs from the Unmanned Space Vehicle Model, 8th Edition (USCM8); and (3) actual cost values for spacecraft subsystems and components whenever such information could be obtained from manufacturers (Boehm, 2000; Tecolote Research Inc., 2009; Tieu, Kropp, \& Lozzi, 2000). In total the cost model employs 31 CERs characterizing a spacecraft's respective nonrecurring (NRE), recurring (RE), and Operations Support (e.g., human labor). The cost model is used to quantify the Static and Dynamic Lifecycle Cost (LCC) of a spacecraft.

3. Dynamic Model

The dynamic (lifecycle cost) model quantifies the Dynamic Lifecycle Cost of a spacecraft subject to lifecycle uncertainties by simulating potential lifecycle's for that spacecraft via a MCA. It is important to recognize the distinction between Static and Dynamic Lifecycle Cost. Static Lifecycle Cost only accounts for the cost of developing, launching, and operating a spacecraft. Therefore, Static Lifecycle Cost is the lower-bound lifecycle cost for a given spacecraft since it does not account for the adverse lifecycle cost implications of lifecycle uncertainties. In contrast, for a given spacecraft, the Dynamic Lifecycle Cost quantifies the totality of the Static Lifecycle Cost, and in addition, accrues the RE and launch costs associated with replenishing spacecraft/modules throughout the lifecycle due to risks resulting from lifecycle uncertainties (e.g., on-orbit failure).

\section{SET Outputs}

The SET outputs form the remaining functional division of the SET. There are four levels of detail (fidelity) quantified by the SET outputs; from highest to lowest fidelity, these are designated as component, subsystem, module, and system-level outputs respectively. Each successively lower fidelity-level output aggregates the characteristics of the higher fidelity-level outputs; therefore, system-level outputs aggregate the characteristics of the outputs from its respective modules, module-level outputs in turn aggregate the characteristics of the outputs from its respective subsystems, and so forth.

The system-level SET outputs are preceded by the word System and include metrics such as System Static Lifecycle Cost, Mass, and Propellant Usage. The module-level SET outputs are preceded by the name of the module they correspond to and include metrics such as (Payload) Module Dynamic Lifecycle Cost, Mass, and Propellant Usage. The subsystem-level SET outputs are preceded by the name of the subsystem they correspond to and include metrics such as Thermal Control Subsystem (TCS) size/volume, Communications mass, and RSM Payload cost. And the component-level SET outputs are referred to by the specific names of components they characterize and include metrics such as RSM Payload Mirror heat dissipation, Propellant Tank length/radius/wall thickness, and Reaction Wheel Unit ( $R W U$ ) electrical power consumption and spin rate (i.e., angular velocity).

For a given spacecraft architecture and its respective SET Lifecycle \& Design and Launch Vehicle inputs, the total number of outputs produced by the SET can be determined by Eq. (1). Note that all the SET outputs are candidate metrics for populating the value proposition corresponding to a given spacecraft.

$$
\text { Outputs }_{\text {SET }}=30+n \cdot 105+m \cdot[(n \cdot 4)+3]
$$

Here Outputs ${ }_{S E T}$ is the number of outputs produced by the SET, $n$ is the number of modules in a spacecraft, and $m$ is the number of MCA trials employed. For example, Eq. (1) yields 37,845 outputs for a three-module fractionated spacecraft architecture assessed with 2,500 MCA trials. Each of these 37,845 outputs quantifies a physical or a cost related characteristic of the fractionated spacecraft at the component, subsystem, module, or system SET output level. Therefore given the breadth and depth of the SET outputs for a given spacecraft architecture, as is evident by Eq. (1), the SET outputs provide a physical instantiation all three research contributions, namely, the ability to explore value propositions both breadth and depth using cardinal, "traditional" measures of effectiveness. 


\section{Analysis: Spacecraft Evaluation Tool Case Study}

To demonstrate some the SET capability in assessing the value propositions for pointing-intensive, monolithic and fractionated spacecraft performing RSMs, a case study was conducted. The emphasis of the case study is to explore the implications of nuances (differences) in various fractionated spacecraft architectures with regard comparable monolithic spacecraft on the basis of their respective value propositions. The architectural nuances considered include the number of modules, use of shared resources, payload performance, and inter-module separation distance. The case study presentation begins by defining the value proposition and the specific SET input values used for the case study.

\section{A. Spacecraft Value Propositions}

The value proposition must be tractable in the sense that meaningful comparisons between two or more value propositions can readily be made - something accomplished through using fewer, rather than more of the (tens of) thousands of metrics (i.e., SET outputs) quantified for a given spacecraft. Based on this tradeoff, the value proposition for this case study is comprised of three metrics: System Static LCC, Dynamic LCC, and Mass.

\section{B. SET Inputs: Launch Vehicle and Lifecycle \& Design}

In terms of the Launch Vehicle group of inputs, of the 22 launch vehicles available for use, 14 were selected as candidate launch vehicles for the dynamic lifecycle simulations of spacecraft. The launch vehicle breakdown is as follows: 1 heavy (United States), 2 large (Russia \& Ukraine, India), 6 medium (United States, Russia), and 5 small (United States, Russia). And in terms of Lifecycle \& Design inputs, only those inputs that drive the results and are subsequently valuable for discussion purposes are enumerated herein. The Lifecycle \& Design inputs that were held constant in the case study are as follows: orbit altitude $(700 \mathrm{~km})$; orbit inclination $\left(98^{\circ}\right)$; mission lifetime (7 years); pointing tolerance $\left(1 \mathrm{e}-4^{\circ}\right)$; average number of in-situ slews per orbit (12); the maximum magnitude of each slew $\left(90^{\circ}\right.$, net); number of MCA trials $(2,500)$; probability of infant mortality $(1.5 \%)$; maximum number of launch vehicles allowed for simultaneous deployment of spacecraft/modules (3); and all lifecycle uncertainties are considered (i.e., launch, technical, environmental, and operational). Given the objectives of the case study, two Lifecycle \& Design inputs were varied, these are the inter-module separation distance $(20,1000,5000 \mathrm{~m})$ and payload ground resolution $(0.5,1,5$, and $30 \mathrm{~m})$.

\section{SET Inputs: Spacecraft Architecture}

The remaining SET inputs define the monolithic and fractionated spacecraft architectures assessed in the case study. Thirteen spacecraft architectures were considered for this case study: 1 monolithic and 12 fractionated. The three-module fractionated spacecraft depicted in Figure 3 employs all shared resources, that is, Comm_CS_C\&DH, ADS_GNS, and Power. Therefore, this architecture provides an excellent example for understanding the subsystem and module composition nuances in all 13 spacecraft architectures assessed in this case study (see Figure 4). Each spacecraft architecture has an identification number; for the architecture represented in Figure 3, its identification number is 26

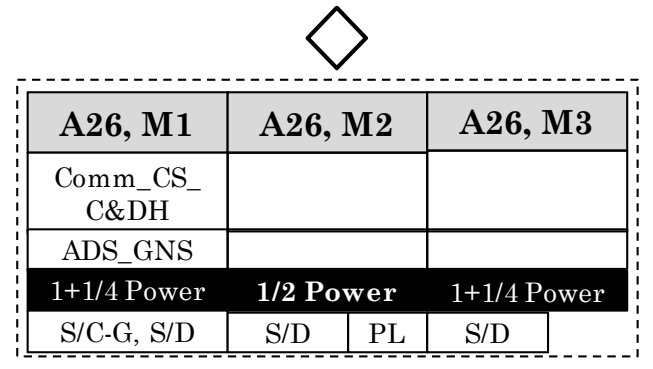

Figure 3. Three-Module Fractionated Spacecraft (Arch 26)

Notation: small directional antenna for intermodule communication (S/D), RSM payload (PL) and is therefore referred to as "Arch 26." Arch 26 has three modules, each of which must have the following subsystems as these are cannot be shared: tracking, telemetry, and control (TT\&C); attitude control system (ACS); guidance control system (GCS); propulsion; thermal control system (TCS); structures; and wiring. Subsequently, these subsystems are not explicitly called out in Figure 3. In Arch 26, module (M1) is the only source for the Comm_CS_C\&DH and ADS_GNS shared resource and module 2 (M2) and 3 (M3) are the recipients of this shared resource. Therefore, M1 must have a dedicated S/C-G antenna as well as two small directional antennas for simultaneous inter-module communication with M2 and M3. And in turn, M2 and M3 must each have one small directional antenna for inter-module communication. In terms of sharing the Power resource, M2 produces and stores only $50 \%(1 / 2)$ of the power that it requires thereby requiring M1 and M3 to each produce and store $100 \%$ (1) of the power they require as well as produce and store $25 \%(1 / 4)$ of the power required by M2. Additionally, the only RSM payload is located on M2 making it the payload module and M1 and M3 infrastructure modules. For all spacecraft considered in the case study, there is only ever one S/C-G antenna and RSM payload employed and these are always located on the first and second modules in a spacecraft respectively; the exception being the monolith (Arch 1) which has both the S/C-G antenna and RSM payload located on the first (and only) module. 


\section{Case Study Spacecraft Architectures: Complete Set}

The set of all 13 spacecraft architectures considered in the case study can be completely differentiated from one another by their respective number of modules and use of shared resources, as is shown in Figure 4. The reason being that for a given spacecraft architecture, the subsystem, RSM payload, and S/C-G antenna composition of the respective modules in the spacecraft follows the pattern observed in Figure 3 by analogy.

Hereafter in the results, each spacecraft architecture is represented by its respective identification number as well as certain color shape, as depicted in Figure 4. The color and shape signify the number of modules and use of shared resources in a spacecraft architecture respectively. Spacecraft architectures 2, 8, and 20 do not employ shared resources and subsequently serve as a necessary relative point of comparison for assessing the purported costs and benefits of fractionation derived from sharing subsystem resources.

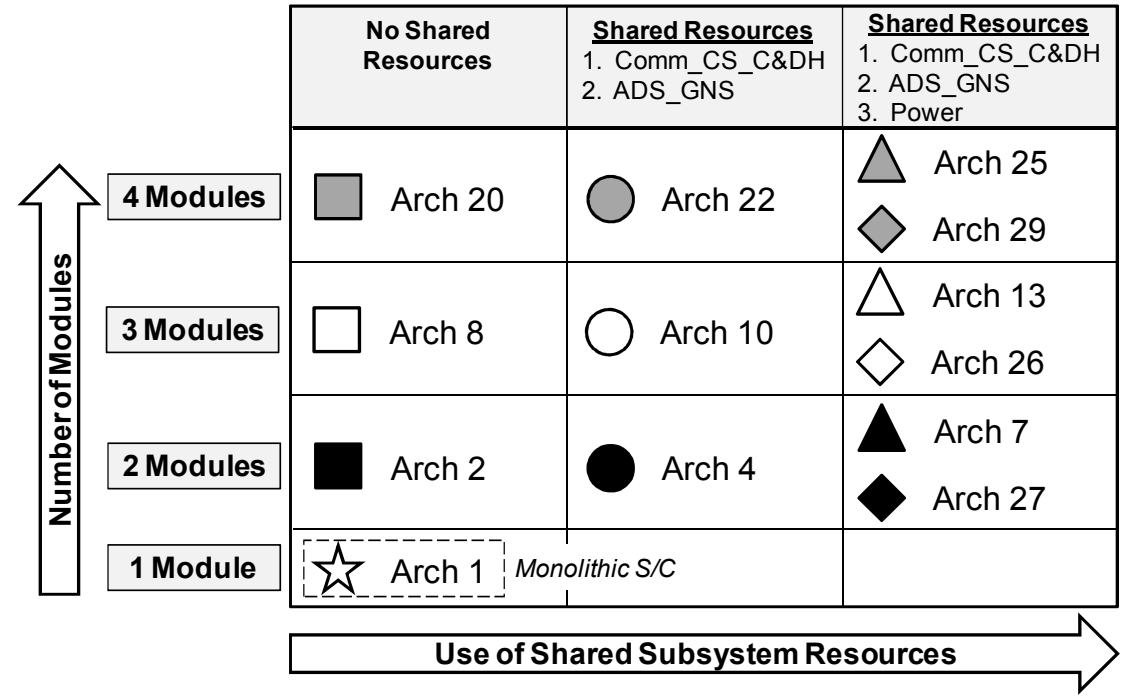

Figure 4. Case Study Spacecraft Architectures.

\section{Results Format}

There are two aspects regarding the manner in which the results are formatted/presented for this case study that require discussion.

\section{Lines of Constant Architecture}

Moving from left to right along the line of data points corresponding to a given spacecraft architecture (with respect to the $\mathrm{x}$-axis value), corresponds to the architecture with a 20,1000, and $5000 \mathrm{~m}$ inter-module separation distance respectively, in Figure 6 and Figure 7; or a 0.5, 1, 5, and $30 \mathrm{~m}$ ground resolution respectively, in Figure 8. For a given spacecraft architecture in Figure 6 and Figure 7, if no line can be discerned it signifies that its respective $\mathrm{x}$ and $\mathrm{y}$-axis values are (or are close to) independent of inter-module separation distance.

\section{LCC Uncertainty}

For a given spacecraft architecture, the MCA analysis in the SET yields 2,500 System Dynamic LCC values that always form a multimodal distribution. Subsequently, the Dynamic LCC measure of central tendency reported in the results is the Median Dynamic LCC. Additionally, there are two sources of uncertainty associated with the Median Dynamic LCC that need to be quantified (O'Neill, 2009). The first source, called cost model uncertainty (CMU), results from statistical uncertainty in the SET cost model, specifically from the CERs. And the second source, called MCA uncertainty (MCAU), is due to the distribution (variance) of Dynamic LCC values resulting from the MCA. Both of these uncertainties in the Median Dynamic LCC value, for a given spacecraft architecture, are best represented relative to an order statistic, five-number summary as is shown in Figure 5.

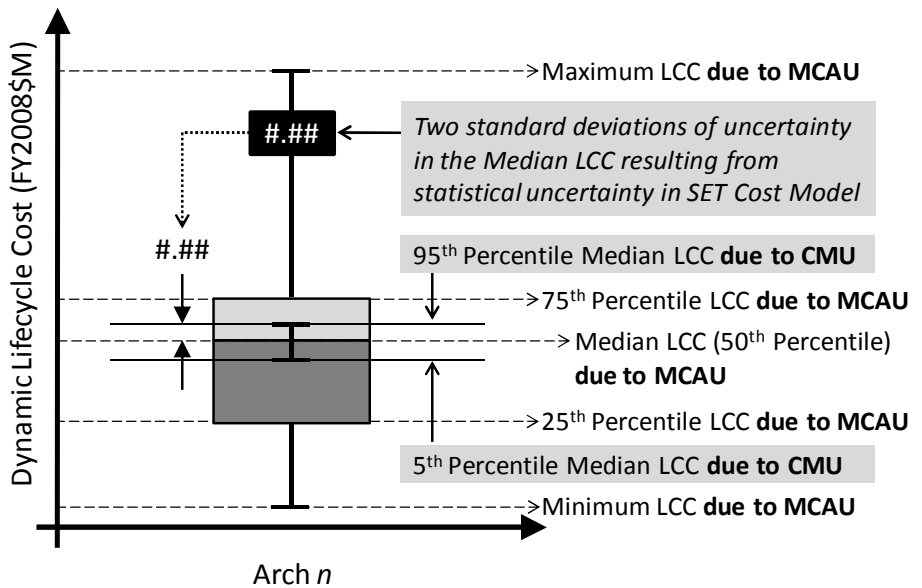

Figure 5. Quantifying Dynamic LCC Uncertainty via an Order Statistic, Five-Number Summary (aka Box-and-Whisker Plot). Notation: uncertainty in Median Dynamic LCC (1) resulting from the SET cost model uncertainty (CMU) and (2) due to the MCA (MCAU) 


\section{E. Implications of Spacecraft Architectures and Inter-Module Separation Distance}

The first constituent of the case study explores of the effect of inter-module separation distance for spacecraft value propositions. With regard to separation distance, Figure 6 and Figure 7 provide the System Static and Dynamic LCC, respectively versus System Mass for all 13 spacecraft architectures shown in Figure 4.

1. Static LCC Trends

Notable trends in Figure 6 include: (1) the two-module architecture, Arch 4, which shares the Comm CS C\&DH and ADS_GNS shared resources has a nearly equivalent Static LCC to that of a comparable monolith; (2) the relative LCCMass trends across classes of architectures (e.g., two-module) are appreciably similar; (3) comparable architectures (e.g., Arch 27, 26, and 29), from least to most expensive, will always correspond to the two, three, and four-module architecture respectively; (4) Arch 2, 8, and 20 are not necessarily the least expensive architectures, thereby enumerating the reduction in LCC gained from sharing certain subsystem resources; and (5) within a given class of architectures, the architectures that do not share any resources will always be the least massive, and consequently as the use of shared resources increases, the Mass tends to increase.

2. Dynamic LCC Trends Notable trends in Figure 7 include: (1) at 20 and $1000 \mathrm{~m}$ separation distances, as compared to the other twomodule architectures, Arch 7 has a significantly higher LCC; (2) for architectures sharing Power, their Mass and Dynamic LCC uniformly increases with regard to separation distance and amount of power sharing
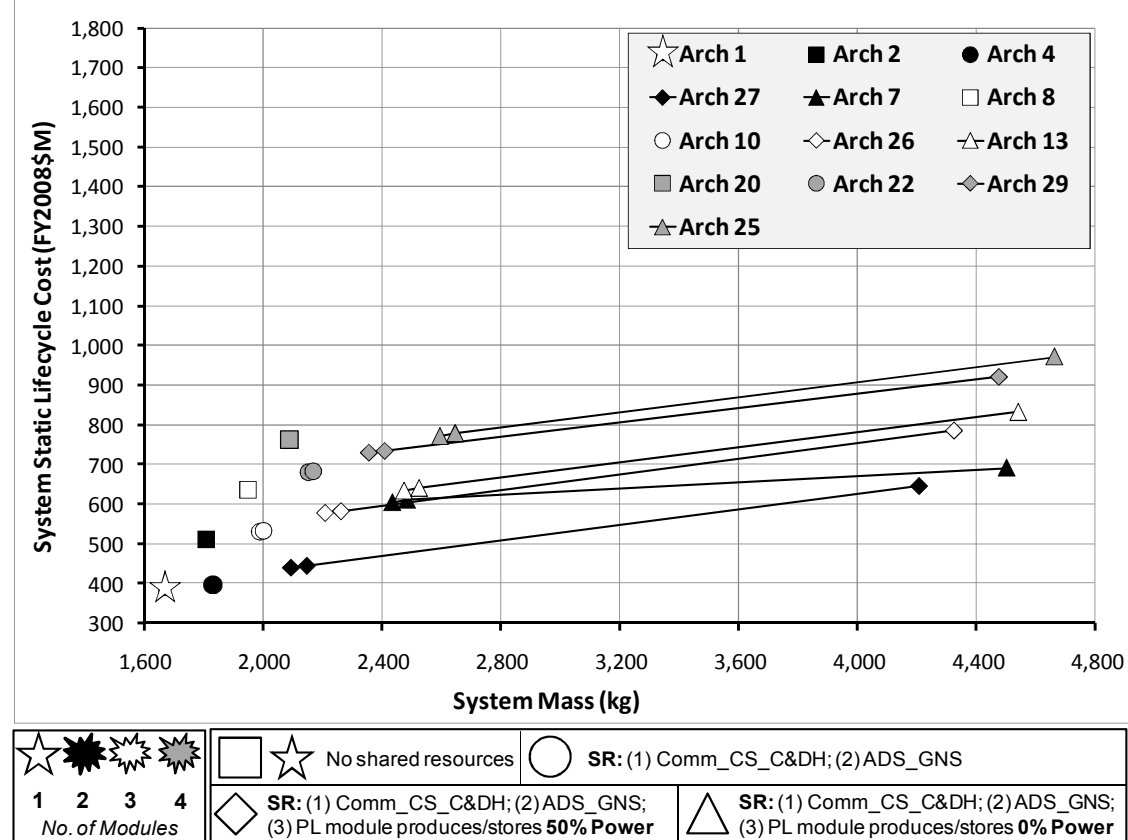

Figure 6. Implications of Separation Distance (Static LCC).

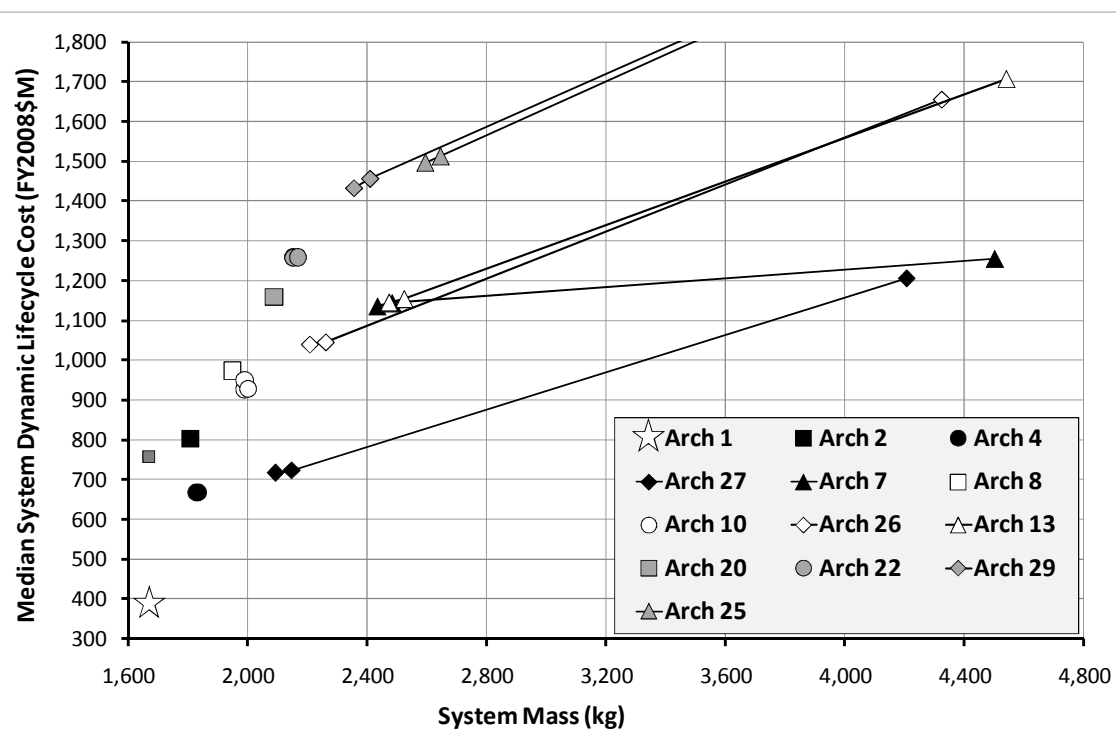

Figure 7. Implications of Separation Distance (Dynamic LCC). (i.e., power not produced and stored by the Payload Module). Subsequently, this case study provides a context in which sharing the Comm_CS_C\&DH and ADS_GNS resources (but not Power) is the most LCC and Masscompetitive use of shared resources to employ in fractionated spacecraft.

3. Static and Dynamic LCC Trends

Notable trends across Figure 6 and Figure 7 include: (1) the Mass is independent of LCC; (2) the Static and Dynamic LCC-Mass trends for two-module architectures are appreciably similar; (3) the Static LCC is much less than the Dynamic LCC ( $\max$ of $58 \%$, min $44 \%$ ); (4) as the number of modules decreases, fractionated architectures become more LCC-competitive to the comparable monolith with regard to Dynamic than Static LCC; and (5) Static LCC fails to appropriately quantify the LCC implications of employing shared resources in fractionated spacecraft, and their respective ability to decouple the pointing-intensive payload and subsystems from other subsystems. 


\section{F. Implications of Spacecraft Architectures and Payload Performance}

The second constituent of the case study explores the effect of payload performance for spacecraft value propositions. Figure 8 provides the Median System Dynamic LCC versus System Mass for 7 of the 13 spacecraft architectures shown in Figure 4 (architectures that do not share resources are not considered). Notable trends in Figure 8 include: (1) two-module architectures are less expensive than the comparable monolith at 0.5 and $1 \mathrm{~m}$ resolutions by up to 90.73 and 94.35 \$M respectively; (2) in transitioning from higher to lower performance payloads (i.e., ground resolution decreases), the LCC-competiveness of the fractionated spacecraft architectures decreases
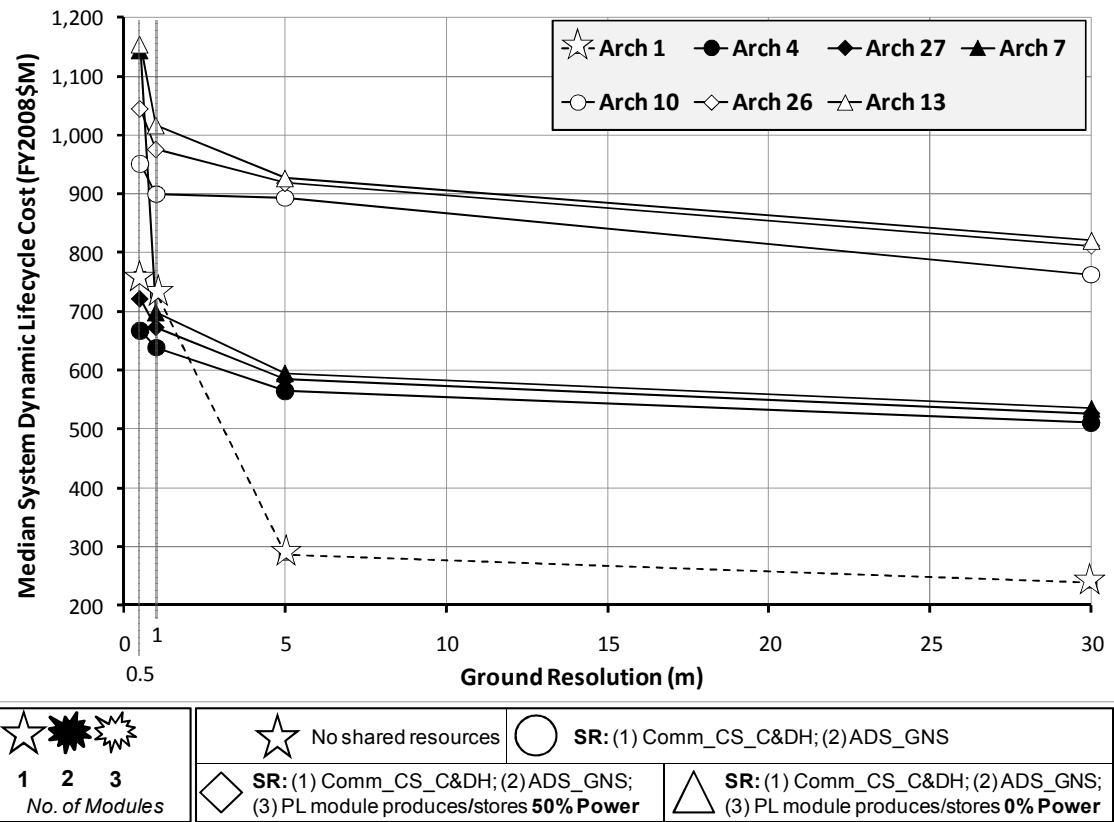

Figure 8. Implications of Payload Performance (Dynamic LCC).

appreciably; (3) regardless of ground resolution, three-module architectures are consistently more expensive than comparable two-module architectures and the monolith; and (4) unlike all the other fractionated spacecraft architectures, Arch 7 and the monolith see a significant decrease in LCC in transitioning from a payload with a 0.5 to $1 \mathrm{~m}$, and 1 to $5 \mathrm{~m}$, ground resolution respectively.

\section{G. Confidence in the Median System Dynamic LCC}

Based on the results shown in Figure 7 and 8 , the suppliant question is; with what level of confidence can it be concluded that certain fractionated spacecraft are less expensive than the comparable monolith? In response, Figure 9 presents the uncertainty (i.e., lack of confidence) in the Median System Dynamic LCC for the monolithic and four most LCCcompetitive fractionated spacecraft in Figure 7.

The comparison of the spacecraft architectures in Figure 9 yields several important insights: (1) the inter-quartile $\left(25-50^{\text {th }}\right.$ percentile) range and $\max / \min$ Dynamic LCC values of Arch 4 and 27 fall within the inter-quartile range and $\mathrm{max} / \mathrm{min}$

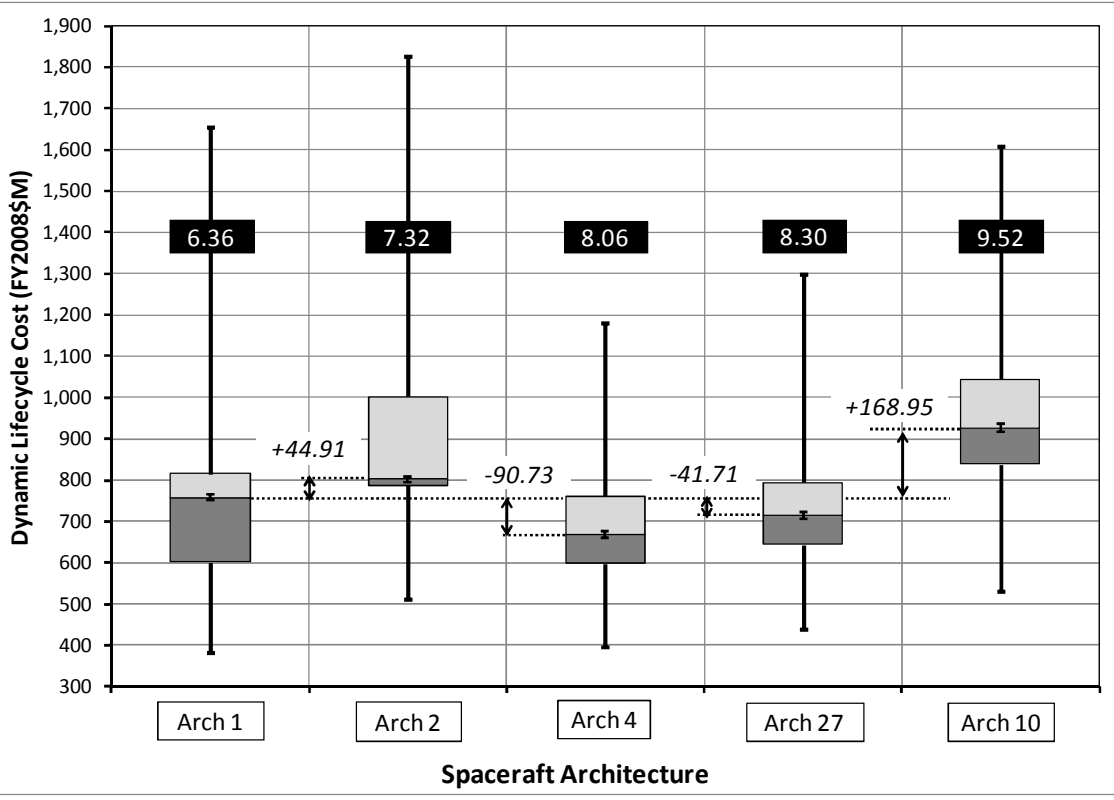

Figure 9. Confidence in Median System Dynamic LCC.

Dynamic LCC values of the monolithic architecture, thereby providing one instantiation of fractionated spacecraft having a lower LCC "risk" (i.e., uncertainty) than a comparable monolith (Arch 1); (2) given the monolithic spacecraft, it can be concluded that comparable two-module fractionated spacecraft can have a lesser Dynamic LCC by up to $104.41 \$ \mathrm{M}$, this accounting for all LCC-related uncertainties; and (3) the $75^{\text {th }}$ percentile Dynamic LCC of the two-module fractionated spacecraft, Arch 4, is about equal to the monolith's $50^{\text {th }}$ percentile LCC. 


\section{Synthesis: Case Study Discussion}

The formation of value propositions for various fractionated spacecraft architectures in the case study provided an instantiation of the engenderment of the three research contributions as well as demonstrated some of the SET capability. The discussion pertaining to the case study results was intended only to enumerate the more obvious trends in the results. Subsequently, the focus of the synthesis is to explore the fundamental reasons as to the trends elicited in the Analysis with regard to monolithic and fractionated spacecraft value propositions.

\section{A. System (Aggregated) Mass and Shared Subsystem Resources}

Fractionated spacecraft will always have a larger aggregate (i.e., all of the modules) mass (and physical size) than a comparable monolithic spacecraft. The reason as to the consistently larger mass of fractionated spacecraft is that they have a higher system-wide redundancy. System-wide redundancy is defined as the total number of a given subsystem present in a fractionated spacecraft relative to one, as a monolith only has one of these subsystems. Consequently system-wide redundancy has a mass (and size) penalty associated with it, manifested in the form of mass and structural overhead, that is, the extra mass and size incurred when replacing one subsystem with a set of smaller subsystems, holding functionality roughly constant. Therefore, for a given subsystem, due to the mass and structural overhead associated with that subsystem present in each of the $n$ modules in a fractionated spacecraft, the aggregate mass (and size) of those $n$ subsystems will be larger than the respective mass and size of that subsystem on the monolith. And in extrapolating this logic to every subsystem in a spacecraft, the aggregate mass of a fractionated spacecraft, on the basis of its respective subsystems, will always be larger than that of a comparable monolith. Subsequently, as the number of modules increases, it only further increases the mass disparity between fractionated and monolithic spacecraft. And in terms of shared resources, as more shared resources are employed, the aggregate mass of a fractionated spacecraft tends to increase; this being due to the hardware required for modules in a fractionated spacecraft acting as shared resource sources and recipients ${ }^{4}$.

\section{B. NRE and RE Costs}

Consequently, the consistently larger aggregate mass of fractionated spacecraft relative to that of a comparable monolithic spacecraft leads to a consistent trend with regard to System Static and Dynamic LCC. An appreciable portion of the Static and Dynamic LCC corresponding to a given spacecraft is due to NRE and RE costs, both of which correlate positively with mass. The NRE costs are incurred only for the initial spacecraft development, but in contrast, the RE costs are incurred for the initial spacecraft development and for every replenishment of the spacecraft and its respective modules throughout the lifecycle. Therefore, the larger aggregate mass of fractionated spacecraft, due to a larger number of modules and/or use of shared resources, has the effect of increasing the Static and Dynamic LCC of fractionated spacecraft relative to that of a comparable monolith (based on NRE and RE costs only). And if the aggregate mass, and hence NRE and RE cost disparity between comparable monolithic and fractionated spacecraft is large enough, it can ensure the Static and Dynamic LCC of fractionated spacecraft will be larger, regardless of the differences between their respective aggregate number and cost of replenishments.

\section{Number and Cost of Lifecycle Replenishments}

As the number of modules and/or use of shared resources increases in a fractionated spacecraft, not only does the aggregate mass tend to increase as was discussed previously, but the number of inter-module dependencies in a fractionated spacecraft increase as well. For fractionated spacecraft, the number of inter-module dependencies increases with an increasing number of modules, holding the use of shared resources constant. As such, intermodule dependencies exacerbate the implications of modules failing on-orbit because a dependent module (i.e., shared resource recipient) will fail if a module it is dependent upon (i.e., shared resource source) fails. Therefore, in terms of aggregate number of replenishments, the implication of an increase in inter-module dependencies in a spacecraft is to increase the aggregate number of replenishments. Consequently, this may increase the number of launch vehicle failures due to the provision of more opportunities in which launches, and thus launch failures, can occur. However in contrast, in terms of aggregate replenishment costs (comprised of RE and launch vehicle costs), relative to the aggregate replenishment costs of a comparable monolith, as the number of inter-module dependencies in a fractionated spacecraft increases, the fractionated spacecraft may, or may not, have a larger aggregate cost of replenishments. The reason for this is differences in launch vehicle usage, and thus launch costs, between comparable monolithic and fractionated spacecraft.

\footnotetext{
${ }^{4}$ Given the low fidelity of the SET discussion in Section II, trends in mass with respect to shared resources at the component, subsystem, and module level, despite being logically reasoned from the physics-based model, cannot be enumerated herein.
} 


\section{Launch Vehicle Usage and Costs}

Through the creation of modules and sharing of subsystem resources, fractionated spacecraft can potentially make use of a smaller launch vehicle or set of smaller launch vehicles as compared to the size of the single launch vehicle employed by a comparable monolith. This is possible because each of the respective modules in a fractionated spacecraft may be individually smaller in mass and size than a comparable monolith ${ }^{5}$, thereby enabling the modules to potentially fit into any combination of up to three smaller launch vehicles as compared to the single launch vehicle employed by the monolith. And since the cost of launch vehicles tends to decrease with the size of the launch vehicle (i.e., its push mass to LEO), fractionated spacecraft can potentially have lesser launch costs than that of a comparable monolith. Therefore, despite the higher aggregate mass, and often number of replenishments, of fractionated spacecraft relative to that of a comparable monolith, due to the dominance of launch vehicle costs on the initial deployment and replenishment costs, fractionated spacecraft can have an equal or lesser Static and/or Dynamic LCC than the monolith. Therefore, an important attribute of fractionated spacecraft, in terms of Static and Dynamic LCC-competiveness, is their ability to attain potentially lesser launch (vehicle) costs than that of a comparable monolithic spacecraft. Subsequently, this attribute of fractionated spacecraft is the reason as to situations observed in Figure 6 through Figure 9 in which fractionated spacecraft have an equal or lesser Static or Dynamic LCC than that of a comparable monolithic spacecraft.

\section{E. Payload Performance}

At 0.5 and $1 \mathrm{~m}$ ground resolutions, certain two-module fractionated spacecraft are less expensive than the comparable monolith despite their larger aggregate mass and number of replenishments. The reason for this follows from the critical attribute of fractionation cited in the previous paragraph, namely, these fractionated spacecraft have appreciably lesser launch costs than that of the comparable monolith, and this enables them to have a lesser Dynamic LCC. In contrast, for the medium and low resolutions of 5 and $30 \mathrm{~m}$ respectively, the monolith is the least expensive spacecraft by a significant LCC margin. The reason for this being that as the resolution decreases, the payload drives less of the physical design of a spacecraft. Subsequently, using fractionation to separate the pointingintensive payload from the other subsystems incurs proportionally more mass (and size) penalties relative to the monolith; this in turn resulting from the mass and structural overhead associated with system-wide redundancy proportionally increasing with decreasing payload dominance (i.e., ground resolution) ${ }^{6}$. Additionally, as the payload ground resolution decreases, due to the larger mass (and size) penalties incurred by fractionated spacecraft, their respective ability to have lesser launch (vehicle) costs than that of a comparable monolith diminishes or ceases altogether. For the two-module fractionated spacecraft in Figure 8, this situation does occur when the ground resolution is 5 and $30 \mathrm{~m}$ because the respective launch vehicle costs these fractionated spacecraft is equal to that of the comparable monolith. Hence, given this and their higher aggregate mass, the Dynamic LCC of the fractionated spacecraft must be larger than that of a comparable monolithic spacecraft.

\section{Conclusion}

This research investigation was motivated by the hypothesis that fractionated spacecraft may be a suitable, if not "better" alternative to monolithic spacecraft. Arguments refuting this hypothesis are often made on the basis of fractionated spacecraft have undesirable concentrated costs manifested in the form large upfront costs associated with developing and deploying them. Upon reflection of the recognizably small glimpse into the cost (and mass) implications of fractionated spacecraft enumerated through the case study results presented herein, insight can still be gained in terms of a response to this hypothesis as well as the alleged concentrated costs of fractionated spacecraft.

Contrary to most previous assessments of fractionated spacecraft, the results of this research demonstrate that the (System) Static and Dynamic Lifecycle Cost of fractionated spacecraft can be equal and less, respectively, than the Static and Dynamic Lifecycle Cost of comparable monolithic spacecraft. Therefore, a meaningful contribution of this research is providing quantitative instantiations that refute the notion of fractionated spacecraft as always having (significantly) larger lifecycle costs than comparable monolithic spacecraft. And in terms of the alleged concentrated costs of fractionated spacecraft, given that certain fractionated spacecraft are shown in the results to

\footnotetext{
${ }^{5}$ This is true for high-resolution fractionated spacecraft with $1000 \mathrm{~m}$ or less inter-module separation distance- and as the case study showed, these are the most LCC competitive fractionated spacecraft. However beyond this observation, nuances in module mass and size with regard to the case study inputs/parameters, despite being logically reasoned from the physics-based model, cannot be tractably enumerated herein given the fidelity of the SET discussion in Section II.

${ }^{6}$ Although not presented in the case study results, the mass of fractionated spacecraft is always appreciably higher than that of a comparable monolith at $0.5,1,5$, and 30 m ground resolutions. (The logic supplied in Section IV.A is applicable.)
} 
have equal Static Lifecycle Cost values to that of comparable monolithic spacecraft, this provides an instantiation of monolithic and fractionated spacecraft having equivalent concentrated costs. However, for completeness, it should also be noted that this was not always the case because in the results numerous fractionated spacecraft have much larger upfront (concentrated) costs than a comparable monolithic spacecraft, as is evident by their appreciably larger Static Lifecycle Costs than the monolith.

In addition, the results of this research demonstrate that fractionated spacecraft can be more susceptible to onorbit failures throughout their respective lifecycle than monolithic spacecraft, this being apparent by situations in which fractionated spacecraft have significantly larger Dynamic Lifecycle Costs than comparable monolithic spacecraft. These situations therefore provide quantitative instantiations of the higher (Dynamic) Lifecycle Cost and "mission risk" of fractionated spacecraft as compared to monolithic spacecraft, for a given space mission.

In revisiting the hypothesis with regard to whether or not fractionated spacecraft are a suitable, if not "better alternative to monolithic spacecraft in the current spacecraft paradigm, this research provides quantitative evidence that both supports and refutes this hypothesis. Therefore the on the basis of this research, and specifically with regard to lifecycle cost, the most appropriate response to this hypothesis is: fractionated spacecraft can and cannot be a suitable, if not "better" alternative to monolithic spacecraft - it is entirely dependent upon the perspective(s) subjectively chosen for which to form the comparison.

\section{References}

${ }^{1}$ Baldwin, C. Y., \& Clark, K. B. (1997). Managing in an Age of Modularity. Harvard Business Review, 75, 84-93.

${ }^{2}$ Esper, J. (2005). Modular, Adaptive, Reconfigurable Systems: Technology for Sustainable, Reliable, Effective, and Affordable Space Exploration. In American Institute of Physics: Space Technology and Applications International Forum 2005. Albuquerque, New Mexico.

${ }^{3}$ Esper, J., Andary, J., Oberright, J., So, M., Wegner, P., Das, A., et al. (2004). Modular, Reconfigurable, and Rapid Response Space Systems: The Remote Sensing Advanced Technology Microsatellite. In American Institute of Aeronautics and Astronautics: 2nd Responsive Space Conference. El Segundo, California.

${ }^{4} \mathrm{O}$ 'Neill, M. G. (2009). Assessing the Impacts of Fractionation on Pointing-Intensive Spacecraft. SM Thesis, Aeronautics and Astronautics, Massachusetts Institute of Technology.

${ }^{5}$ Mathieu, C., \& Weigel, A. (2005a). Assessing the Flexibility Provided by Fractionated Spacecraft. In American Institute of Aeronautics and Astronautics: Space 2005 Conference and Exposition. Long Beach, California.

${ }^{6}$ Brown, O., \& Eremenko, P. (2006a). The Value Proposition for Fractionated Space Architectures. In American Institute of Aeronautics and Astronautics: Space 2006 Conference and Exposition. San Jose, California.

${ }^{7}$ Mandy, C., Sakamoto, H., Saenz-Otero, A., \& Miller, D. W. (2007). Implementation of Satellite Formation Flight Algorithms using SPHERES Aboard the International Space Station. In 3rd Annual International Symposium on Space Flight Dynamics. Annapolis, Maryland.

${ }^{8}$ McGhan, C. L., Besser, R. L., Sanner, R. M., \& Atkins, E. M. (2006). Semi-Autonomous Inspection with a Neutral Buoyancy Free-Flyer. In American Institute of Aeronautics and Astronautics: Guidance, Navigation, and Control Conference and Exhibit. Keystone, Colorado.

${ }^{9}$ Kerslake, T. W. (2008). Lunar Surface-to-Surface Power Transfer. In University of New Mexico's Institute for Space and Nuclear Power Studies: Space Technology and Applications International Forum. Albuquerque, New Mexico.

${ }^{10}$ Diller, N. P. (2002). Utilizing Multiple Attribute Tradespace Exploration with Concurrent Design for Creating Aerospace Systems Requirements. SM Thesis, Aeronautics and Astronautics, Massachusetts Institute of Technology.

${ }^{11}$ Mathieu, C., \& Weigel, A. (2005b). Assessing the Flexibility Provided by an On-Orbit Infrastructure of Fractionated Spacecraft. In 56th International Astronautical Congress of the International Astronautical Federation. Fukuoka, Japan.

${ }^{12}$ Mathieu, C., \& Weigel, A. (2006). Assessing the Fractionated Spacecraft Concept. In American Institute of Aeronautics and Astronautics: Space 2006 Conference and Exposition. San Jose, California.

${ }^{13}$ Mathieu, C. (2006). Assessing the Fractionated Spacecraft Concept. SM Thesis, Engineering Systems Division and Technology and Policy Program, Massachusetts Institute of Technology.

${ }^{14}$ Ross, A. (2006). Managing Unarticulated Value: Changeability in Multi-Attribute Tradespace Exploration. $\mathrm{PhD}$ Dissertation, Engineering Systems Division, Massachusetts Institute of Technology.

${ }^{15}$ Ross, A. (2003). Multi-Attribute Tradespace Exploration with Concurrent Design as a Value-Centric Framework for Space System Architecture and Design. SM Thesis, Aeronautics and Astronautics, Massachusetts Institute of Technology.

${ }^{16}$ Molette, P., Cougnet, C., Saint-Aubert, P., Young, R., \& Helas, D. (1984). Technical and Economical Comparison Between a Modular and Geostationary Space Platform and a Cluster of Satellites. Acta Astronautica, 12(11), 771-784.

${ }^{17}$ Brown, O. (2004). Reducing the Risk of Large Scale Space Systems Using a Modular Architecture. In The Aerospace Corporation: Space Systems Engineering \& Risk Management Symposium. Los Angeles, California.

${ }^{18}$ Brown, O., \& Eremenko, P. (2008). Application of Value-Centric Design to Space Architectures: The Case of Fractionated Spacecraft. In American Institute of Aeronautics and Astronautics: Space 2008 Conference and Exposition. San Diego, California. 


\footnotetext{
${ }^{19}$ Brown, O., Eremenko, P., \& Roberts, C. (2006). Cost-Benefit Analysis of a Notional Fractionated SATCOM Architecture. In American Institute of Aeronautics and Astronautics: 24th International Communications Satellite Systems Conference (ICSSC). San Diego, California.

${ }^{20}$ Brown, O., Long, A., Shah, N., \& Eremenko, P. (2007). System Lifecycle Cost Under Uncertainty as a Design Metric Encompassing the Value of Architectural Flexibility. In American Institute of Aeronautics and Astronautics: Space 2007 Conference and Exposition. Long Beach, California.

${ }^{21}$ Defense Advanced Research Projects Agency. (2008). DARPA Awards Contracts for Fractionated Spacecraft Program. News Release. Retrieved February 26, 2008, from www.darpa.mil/news/2008/F6.pdf.

${ }^{22}$ Shah, N., \& Brown, O. (2008). Fractionated Satellites: Changing the Future of Risk and Opportunity for Space Systems. High Frontier (United States Air Force), 29-36.

${ }^{23}$ Isakowitz, S. J., Hopkins, J. B., \& Hopkins Jr., J. P. (2004). International Reference Guide to Space Launch Systems: Fourth Edition. Reston, Virginia: American Institute of Aeronautics and Astronautics.

${ }^{24}$ Brown, O., \& Eremenko, P. (2006b). Fractionated Space Architectures: A Vision for Responsive Space. In American Institute of Aeronautics and Astronautics: 4th Responsive Space Conference. Los Angeles, California.

${ }^{25}$ McManus, H., \& Hastings, D. E. (2006). A Framework for Understanding Uncertainty and its Mitigation and Exploitation in Complex Systems. IEEE Engineering Management Review, 34(3), 81-94.

${ }^{26}$ Lack, M. G. (2006). Two-Dimensional Flow Field Simulation through Proper Orthogonal Decomposition and Practical Zooming Methods. In American Institute of Aeronautics and Astronautics: Region I-Northeastern Student Conference. Syracuse University, Syracuse New York.

${ }^{27}$ Boehm, B. (2000). Software Cost Estimation with COCOMO II. Englewood Cliffs, New Jersey: Prentice Hall.

${ }^{28}$ Tecolote Research Inc. (2009). USCM8: Unmanned Space Vehicle Cost Model, 8th Edition. Retrieved June 4, 2009, from https://www.uscm8.com.

${ }^{29}$ Tieu, B., Kropp, J., \& Lozzi, N. (2000). The Unmanned Space Vehicle Cost Model - Past, Present, and Future. In American Institute of Aeronautics and Astronautics: Space 2000 Conference and Exposition. Long Beach, California.
} 